\title{
Color Image Correction of Art Paintings Based on Artists' Color Features and Illuminant Conversion
}

\author{
Yoshie IMAI,**, Ryoichi SAITO***, Takahiko HORIUCHI and Shoji TOMINAGA** \\ *Graduate School of Advanced Integration Science, Chiba University \\ **Toshiba Corporation, Corporate Research \& Development Center \\ ***Faculty of Engineering, Chiba University
}

Received November 5, 2012, Accepted January 21, 2013

\begin{abstract}
This paper proposes a method to correct the color-mismatch images of paintings based on artists' color features, and reproduce the exact color images under a specific illumination condition. First, we describe a standard image database consisting of oil paintings and an algorithm for extracting artists' color features. The color distribution is analyzed by PCA and described with an ellipsoid to represent the standard color features. Next, the color correction is based on the coordinate transformation of pixel values in a color-mismatch image so that the color features are fitted to the artist's color features. We present the correction algorithms using two color spaces of the sRGB and the CIELAB. Moreover, we describe a procedure for rendering the exact color images under a favorite illuminant. The correlated color temperature of scene illuminant is estimated and the color image under D65 is further transformed to a target image. The feasibility of the proposed method to recover the exact color images is examined with color-mismatch image samples of famous oil paintings.
\end{abstract}

KEYWORDS: color image, color correction, art painting, color feature, illuminant estimation, color temperature

\section{Introduction}

Exact color image reproduction of fine-art paintings is an important issue in many fields including digital archiving, color reproduction, and image rendering ${ }^{1)}$. However, most color images of art paintings which people see are the digital image data, taken by scanning the printed matter in picture collection books or obtained by retrieving museum pictures through the internet. In such a case, we should note that color appearance of the reproduced color images does not match to the color appearance of the original paintings observed under a specific museum lighting environment. The color mismatch occurs in the process including transmission system from image acquisition to image display/printing. The problem of color mismatch cannot be solved as a usual color reproduction problem, because the original observation conditions such as the spectral sensitivities of imaging device and the illuminant spectrum are unknown.

It is thought that an artist painter has peculiar use of color. In fact, there is a report that color features of images from a Web museum were investigated to classify the style of fine-art paintings by individual artists ${ }^{2}$. However we suspect that the colors observed with the images in the Web museum are mismatched colors. In general, individual artists have their own features in painting style, color preference, paints, and materials. Therefore, the information of the individual artists' color features is useful for correcting the mismatched color images. For example, we acquire color images from the original paintings of a particular artist by using a calibrated imaging system to extract his/her color features from the captured images. These color features are available for color correction of the color-mismatch images.

So far we had created a standard image database of individual artists by taking the photographs of the famous paintings in several museums using a calibrated digital camera. Then we analyzed the color features of each image data set ${ }^{3}$. The present paper proposes a method to correct the color-mismatch images of paintings based on artists' color features, and reproduce the exact color images under a specific illumination condition. In this paper, we first describe an algorithm for extracting artists' color features. A color histogram is created on a color space to represent the pixel distribution of the standard image data for each artist. Principal component analysis (PCA) is applied to the color histogram to compute eigenvalues and eigenvectors of the dataset. These statistical quantities are regarded as standard 
color features for the artist. These standard color features are compared with the subjective evaluation of impressions of paintings. Next, we calculate the color distribution of a color mismatch image in the same color space and determine the color features in the same way. Then, the latter color features are transformed to be coincident with the standard color features, so that the color distribution of the color mismatch image is close to the standard features inherent to the artist. As a result, color image correction is performed.

Figure 1 shows the flow of the data image processing. The first step is image acquisition of original paintings. We photograph the paintings by using a calibrated camera under different illuminants with a white reference plate to make the standard database under D65. The standard color features are determined from the image database for each artist. Next, color correction of color-mismatch images is performed based on transformation of the color features to the standard color features. The sRGB color space and the CIELAB color space are used for this correction. In addition, illuminant conversion is applied to the corrected image under D65 to reproduce the color images observed in favorite lighting environment. For this purpose, we describe a computation procedure for estimating color temperature of scene illuminant. An exact color image of the painting under the target illuminant is rendered from illumination conversion based on the color temperature and a color adaptation model.

In the following, Section 2 describes the image databases of famous art paintings and the color feature extraction for individual artists. Section 3 develops a color correction algorithm for color mismatch images. Section
4 describes color image rendering under different illumination. Section 5 shows experimental results to demonstrate the feasibility of the proposed method.

\section{Painting Image Databases and Color Feature Ex- traction}

\subsection{Standard samples of art paintings}

A large database collecting high resolution digital images (about $4500 \times 3500$ pixels) of paintings by famous artists was constructed with the photographs taken in the Orsay Museum and the Orangerie Museum in Paris, where photography was permitted. From the dataset, we selected paintings by six artists. The selected artwork paintings by each artist are listed as follows:

\section{Paul Cezanne; 8 works}

(Portrait of Madame Cézanne

In the Park of Chateau Noir

The House of Doctor Gachet in Auvers

Still Life with Onions

Still Life with a Basket (Kitchen Table)

The Hanged Man's House, Auvers-sur-Oise

The Card Players),

\section{Vincent van Gogh; 7 works}

(The Arlesienne

The Church in Auvers-sur-Oise, View from the Chevet

Dr. Paul Gachet

Self-Portrait 1887

Van Gogh's Bedroom in Arles

Thatched Cottages at Cordeville, Auvers-sur-Oise Self-Portrait 1889),

Edouard Manet; 7 works

(The Balcony

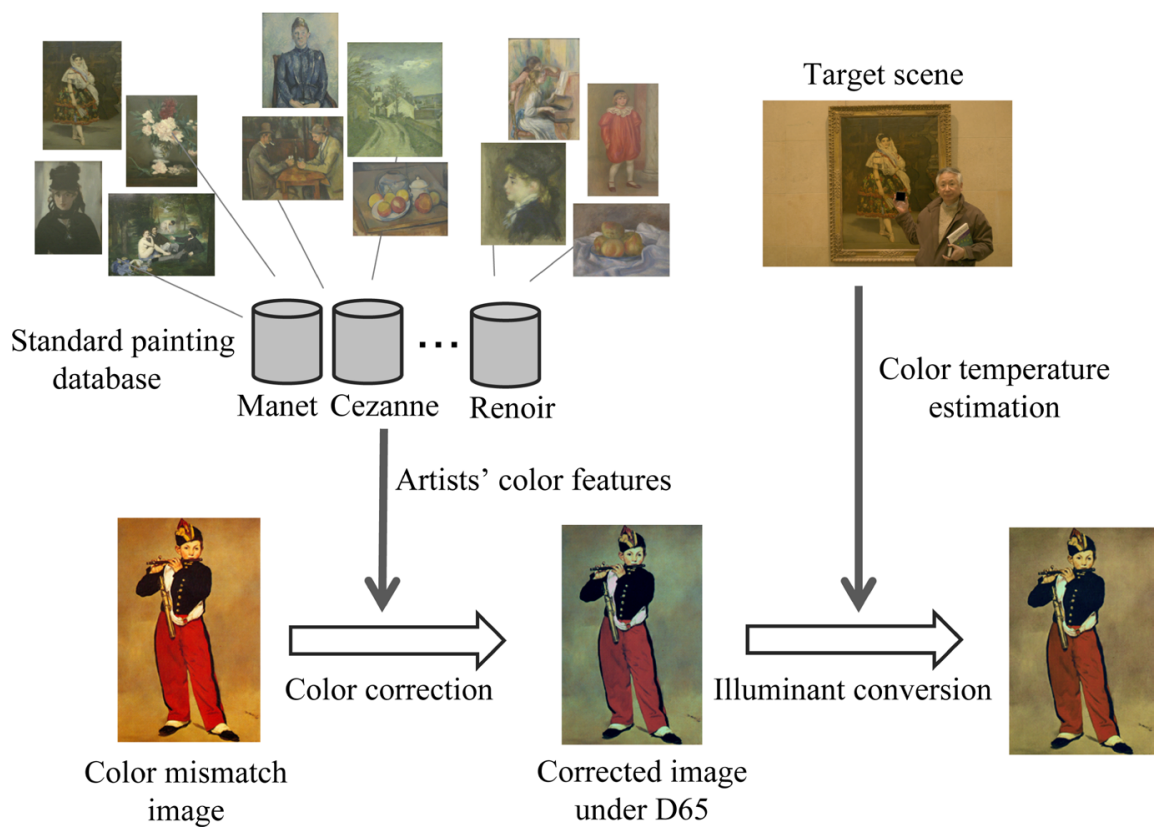

Figure 1 Flow of the data image processing 
The Fife Player

Lola of Valence

Berthe Morisot With a Bouquet of Violets

Olympia

Luncheon on the Grass

Vase of Peonies on a Pedestal)

Jean-Francois Millet; 5 works

(A Winnower

Shepherdess with her flock

Spring

The Angelus

The Gleaners),

\section{Claude Monet; 9 works}

(Wind Effect, Series of the Poplars

Rouen Cathedral

Study of a Figure Outdoors (Facing Left)

Poppies

London, Houses of Parliament. The Sun Shining

through the Fog

The Railway Bridge at Argenteuil

The Saint-Lazare Station

The Argenteuil Bridge

Red Boats at Argenteuil)

Pierre-Auguste Renoir; 12 works

(Apples and Pears

Large Nude

The Bathers

Dance at Le Moulin de la Galette

Girls at the Piano

Claude Renoir in Clown Costume

Portrait of a Lady (Mme Georges Hartmann)

Dance in the Country

Dance in the City

Gabrielle and Jean

Portrait of Margot (Portrait of a Model)

Nude in the Sun

Bather with Long Hair)

\subsection{Imaging system}

We used a calibrated digital RGB camera (Canon EOS 1Ds) with an image resolution of $4992 \times 3883$ pixels and a quantization level of 12 bits. The camera sensitivity functions are shown in Figure 2. We found that a good linear relationship exists between the input luminance values to the camera and the output RGB values from the camera.

The illumination for each painting is different. The illumination sources observed in the two museums were various light bulbs and daylight through transparent glass windows, so that each painting is basically considered to have different light source. In the image capturing, the light source information was recorded for each painting by using a white reference plate as shown in Figure 3. From this light source observation, we discovered that the correlated color temperature of

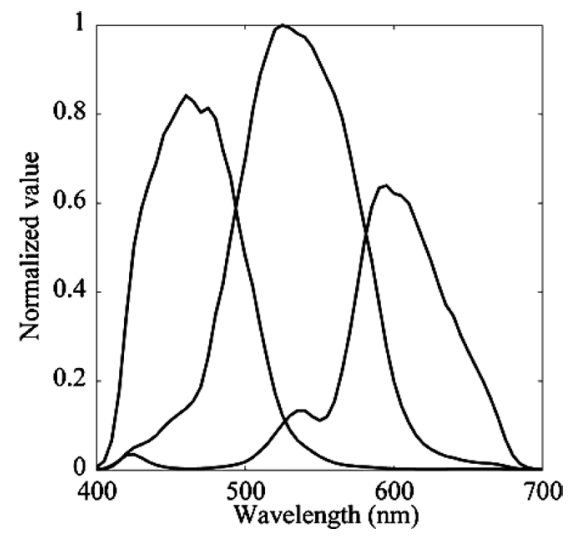

Figure 2 Spectral sensitivity functions of the camera used in this paper

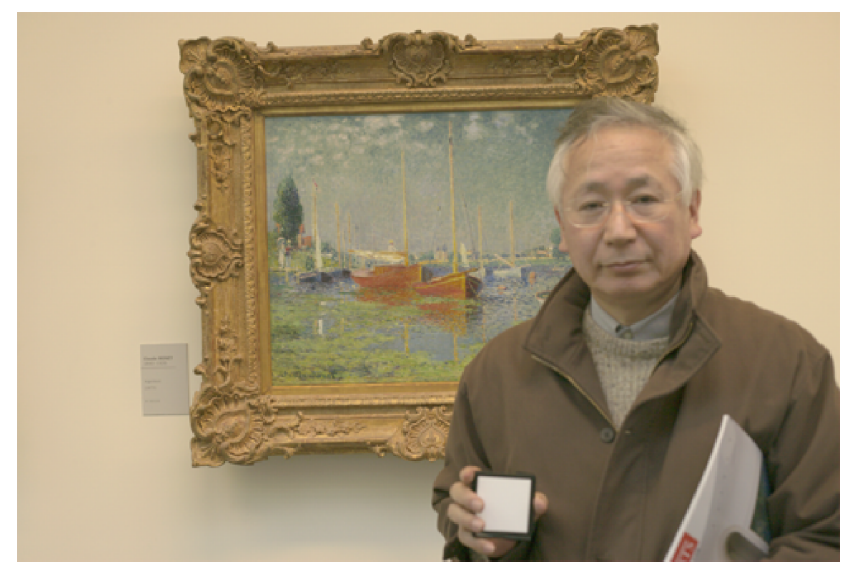

Figure 3 Capturing with a white calibration plate

the light source ranged from $2900 \mathrm{~K}$ to $6300 \mathrm{~K}$.

\subsection{Image transformation into standard light source}

Since the illumination condition is different among the image dataset, the captured RGB images are transformed into the RGB images under the standard light source of D65. This transformation consists of the following two steps:

1. Determination of correlated color temperature illuminating each painting

2. Transformation to an image viewed under D65 illuminant

First, the color temperature can be determined based on the chromaticity coordinates $(\mathrm{x}, \mathrm{y})$ calculated from observations of the white calibration plate.

Second, the image transformation is done using a color temperature conversion method ${ }^{4}$. Let $T$ and $T_{o}$ be the color temperatures of the observation illuminant and the illuminant D65, respectively. The camera RGB values captured at the temperature $T$ can be transformed to the color values $\left(R_{o}, G_{0}, B_{o}\right)$ at the standard temperature $T_{o}$ by a simple equation 


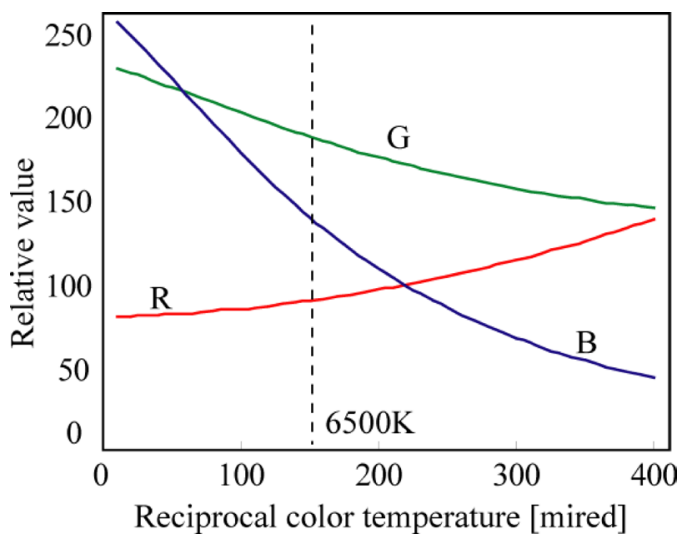

Figure 4 Color temperature variation of RGB values

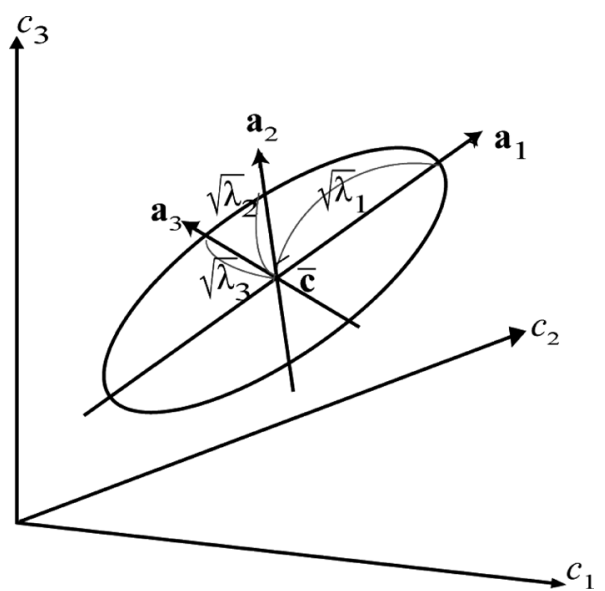

Figure 5 Eigenvalues and eigenvectors representing the features of statistical distribution

$$
\left(R_{o}, G_{o}, B_{o}\right)=\left(w_{R} R, w_{G} G, w_{B} B\right),
$$

where $\left(w_{R}, w_{G}, w_{B}\right)$ are the weighting coefficients. Figure 5 shows the color temperature variation of $\mathrm{RGB}$ values for the present camera. Note that the horizontal axis is represented in the reciprocal color temperature, defined by $M=10^{6} / T$, and the vertical axis is normalized into 8 bits. Three curves of RGB can be calculated using the black body radiator, the camera-spectral sensitivity functions, and the data set of surface-spectral reflectances. The detailed algorithm is presented $\mathrm{in}^{4}$ ). When $\mathrm{T}$ is the color temperature, the weights for conversion to $T_{o}$ are determined as the ratios $\left(w_{R}, w_{G}, w_{B}\right)=\left(R_{o} / R_{T}\right.$, $\left.G_{o} / G_{T}, B_{o} / B_{T}\right)$ of the variation curves in Figure 4.

\subsection{Color feature extraction}

First, the RGB image data under D65 are transformed into XYZ tristimulus values by multiplication of a $3 \times 3$ matrix, which is constructed by fitting the spectral sensitivity functions in Figure 3 to CIE 1931 (2 deg) color matching functions. We use the sRGB color space and the CIELAB color space as the standard for analyzing color features found in images of paintings by different artists. The sRGB color space is commonly used as a standard monitor color space ${ }^{5}$. The calculation of sRGB values from the XYZ tristimulus values is given as

$$
\left[\begin{array}{l}
R \\
G \\
B
\end{array}\right]=\left[\begin{array}{rrr}
3.2406 & -1.5372 & -0.4986 \\
-0.9689 & 1.8758 & 0.0415 \\
0.0557 & -0.2040 & 1.0570
\end{array}\right]\left[\begin{array}{l}
X \\
Y \\
G
\end{array}\right]
$$

The CIELAB color space approximates a perceptually uniform color space, which is defined in terms of the defined in $\mathrm{XYZ}$ as

$$
\begin{aligned}
& L^{*}=116\left(Y / Y_{n}\right)^{1 / 3}-16 \\
& a^{*}=500\left\{\left(X / X_{n}\right)^{1 / 3}-\left(Y / Y_{n}\right)^{1 / 3}\right\}, \\
& b^{*}=200\left\{\left(Y / Y_{n}\right)^{1 / 3}-\left(Z / Z_{n}\right)^{1 / 3}\right\},
\end{aligned}
$$

where $X_{\mathrm{n}}, Y_{n}$ and $Z_{n}$ are the $\mathrm{XYZ}$ tristimulus values of the reference white.

The color histogram of all image data acquired for each artist is created in the above coordinate systems of $(R, G, B)$ and $\left(L^{*}, a^{*}, b^{*}\right)$. The PCA is applied to the color histogram to compute eigenvalues and eigenvectors of the image dataset for each artist. Note that the size and viewing positions of the original paintings are different. Therefore, in our analysis, the size of original paintings is neglected, and the image size of different paintings is normalized to nearly the same size.

The PCA is known as a method for extracting statistical features of a data set in a linear vector space. Let $\mathbf{c}$ and $\overline{\mathbf{c}}$ be the 3D column vector of each color value in a color space and the mean vector over all pixels, respectively. The covariance matrix is then described as

$$
\mathbf{A}=\mathbf{E}\left[(\mathbf{c}-\overline{\mathbf{c}})(\mathbf{c}-\overline{\mathbf{c}})^{\mathrm{T}}\right]
$$

where $\mathbf{E}[\bullet]$ denotes the expectation for the random variable, and $\mathrm{T}$ denotes the matrix transposition. Let $\left(\lambda_{1}, \lambda_{2}, \lambda_{3}\right)$, where $\left(\lambda_{1} \geq \lambda_{2} \geq \lambda_{3}\right)$, and $\left(\mathbf{a}_{1}, \mathbf{a}_{2}, \mathbf{a}_{3}\right)$ be the eigenvalues and the eigenvectors of the matrix $\mathbf{A}$, respectively, which satisfy the basic equations

$$
\mathbf{A} \mathbf{a}_{i}=\lambda_{i} \mathbf{a}_{i} \quad(i=1,2,3) .
$$

The eigenvalues and eigenvectors represent the features of the statistical distribution as shown in Figure 5. The eigenvectors correspond to the orthogonal directions of the ellipsoid to represent the distribution, and the square roots of the eigenvalues correspond to their lengths. Therefore, $\left(\mathbf{a}_{1}, \lambda_{1}\right)$ is the first principal component, where $\mathbf{a}_{1}$ determines the direction of the main axis and $\lambda_{1}$ is the variance along the main axis.

The eigenvectors and eigenvalues are regarded as the principal directions of color distribution and the deviations from the average color, respectively. The average color, the color distribution directions, and the color deviations characterize the color distribution in a color space for each painting. Suppose that we collect many 
paintings by individual artists, and compute the average color, the color distribution directions, and the color deviations for the sets of the individual artist's paintings. From a statistical point of view, the three quantities can be considered to depend on the individual artists. Therefore, the average color, eigenvectors, and eigenvalues represent the color features of the individual artists.

As an example, Figure 6(a) demonstrates the color distribution of the image set in the sRGB space, which consists of six paintings by Manet. The PCA is applied to the color distribution. Figure 6(b) shows the ellipsoid approximation of the color distribution in Figure 6(a). A comparison between Figure 6(a) and 6(b) suggests that the color distribution can be represented effectively by an ellipsoid, and the three quantities of the average color, the eigenvectors, and the eigenvalues represent the color features of Manet's paintings. Figure 7 shows the ellipsoids obtained from the respective image datasets of the six artists in sRGB color space.

Figure 8 shows the corresponding ellipsoids in the CIELAB color space. Table 1 lists the numerical values of color features for all artists in the CIELAB color space. It should be noted that the positions and the orientations of the ellipsoids differ for every artists. The position is an important feature related to lightness and chromaticity. The size represents the variation of color distribution.

Consider van Gogh's color features. Van Gogh's ellipsoid is placed at an upper position and large in the

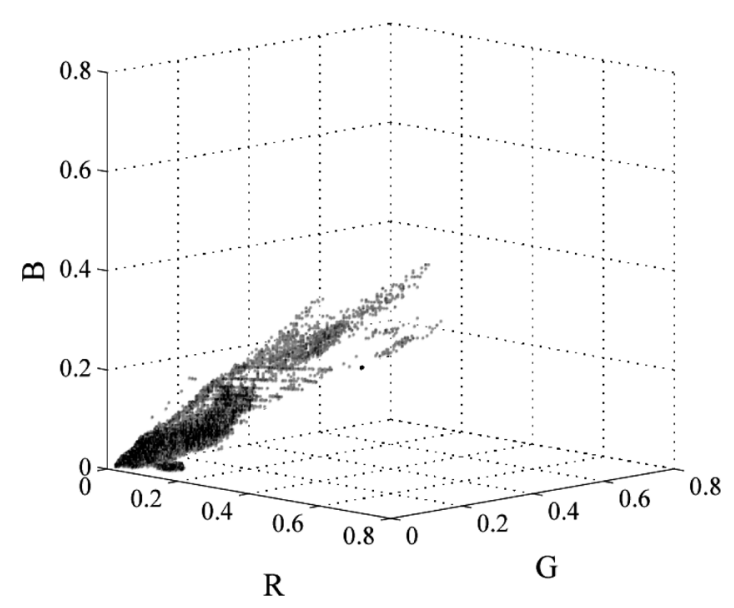

(a) Color distribution

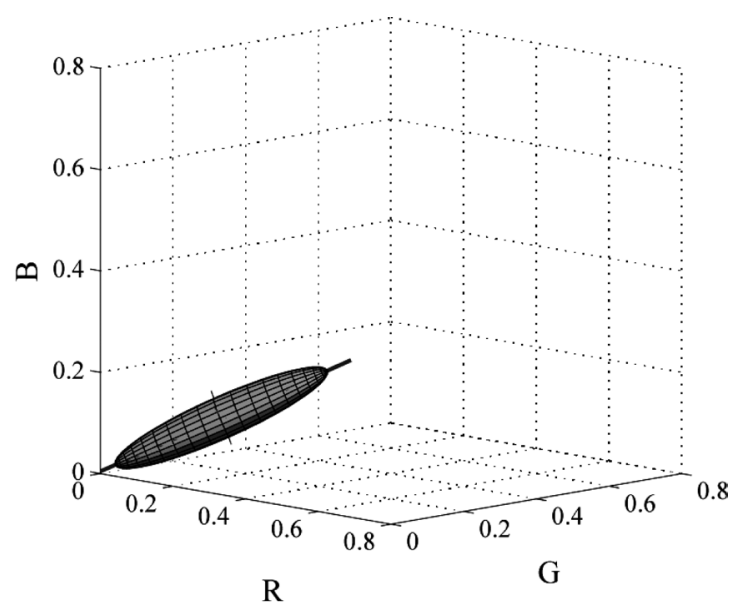

(b) Ellipsoid

Figure 6 Color distribution of image data for Manet

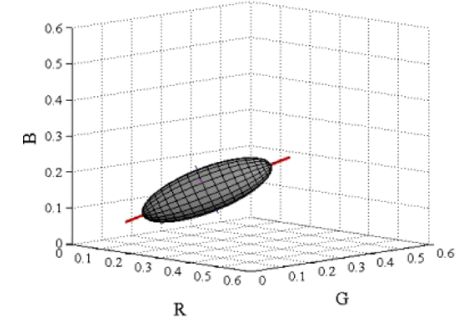

(a) Cezanne

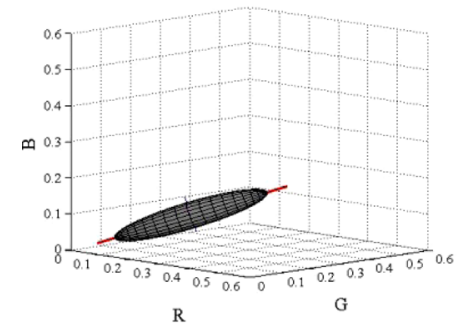

(d) Millet

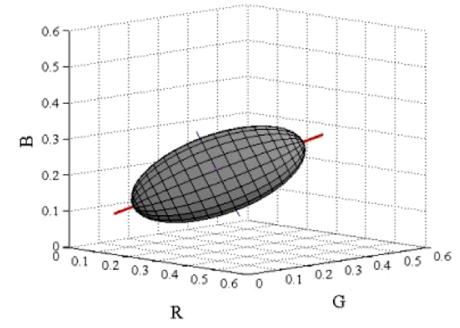

(b) van Gogh

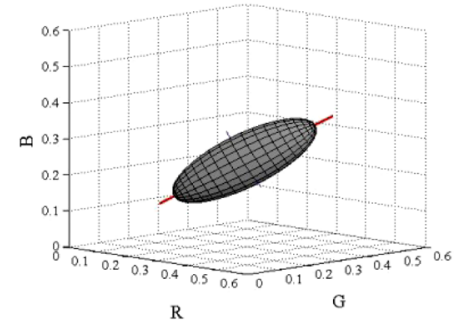

(e) Monet

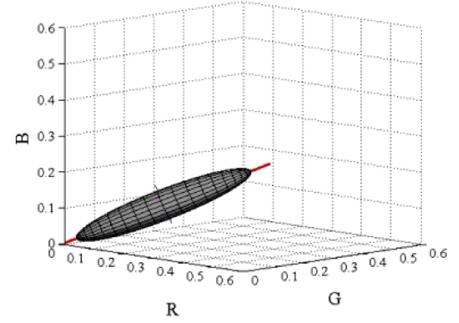

(c) Manet

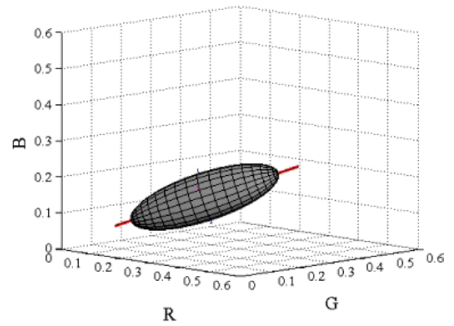

(f) Renoir

Figure 7 Ellipsoids for the image datasets of six artists in the SRGB color space under D65 


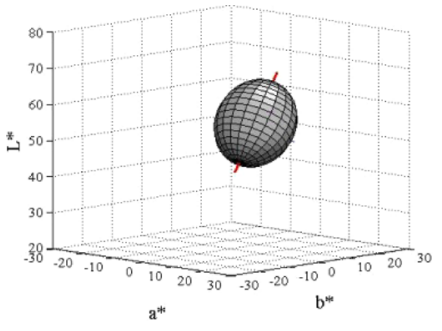

(a) Cezanne

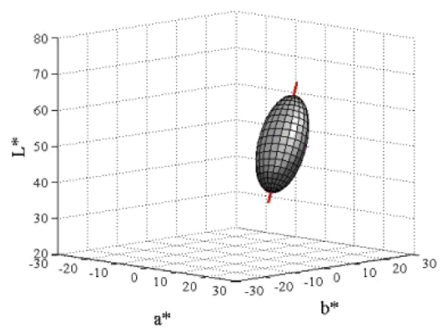

(d) Millet

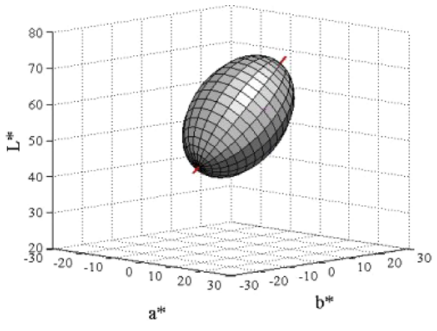

(b) van Gogh

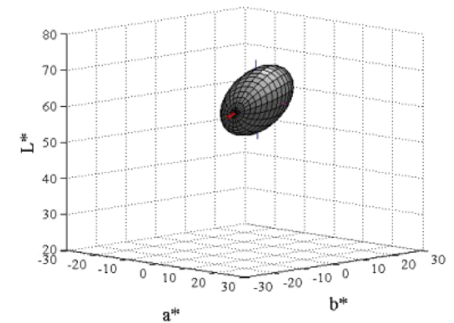

(e) Monet

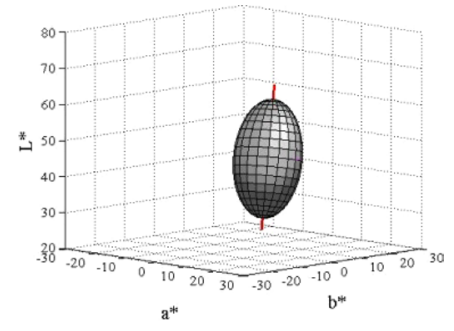

(c) Manet

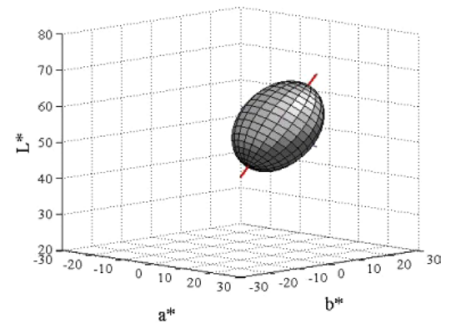

(f) Renoir

Figure 8 Ellipsoids for the image datasets of six artists in the CIELAB color space under D65

Table 1 Artists' color features in the CIELAB color space under D65

\begin{tabular}{|c|c|c|c|c|c|c|}
\hline Artist & & Average Vector & & value & & Eigenvector \\
\hline \multirow{3}{*}{ Cezanne } & \multirow{3}{*}{$\overline{\mathbf{c}}$} & \multirow{3}{*}[52.311,-4.156,11.884]{$^{\mathrm{T}}$} & $\lambda_{1}$ & 162.25 & $\mathbf{a}_{1}$ & {$[0.774,-0.245,0.584]^{\mathrm{T}}$} \\
\hline & & & $\lambda_{2}$ & 75.10 & $\mathbf{a}_{2}$ & {$[-0.588,0.067,0.806]^{\mathrm{T}}$} \\
\hline & & & $\lambda_{3}$ & 35.18 & $\mathbf{a}_{3}$ & {$[0.237,0.967,0.093]^{\mathrm{T}}$} \\
\hline \multirow{3}{*}{ van Gogh } & \multirow{3}{*}{$\overline{\mathbf{c}}$} & \multirow{3}{*}[54.721,-5.038,8.455]{$^{\mathrm{T}}$} & $\lambda_{1}$ & 409.76 & $\mathbf{a}_{1}$ & {$[0.57,-0.235 .0 .788]^{\mathrm{T}}$} \\
\hline & & & $\lambda_{2}$ & 143.62 & $\mathbf{a}_{2}$ & {$[-0.796,0.079,0.6]^{\mathrm{T}}$} \\
\hline & & & $\lambda_{3}$ & 37.01 & $\mathbf{a}_{3}$ & {$[0.203,0.969,0.143]^{\mathrm{T}}$} \\
\hline \multirow{3}{*}{ Manet } & \multirow{3}{*}{$\overline{\mathbf{c}}$} & \multirow{3}{*}[43.192,-3.087,10.412]{$^{\mathrm{T}}$} & $\lambda_{1}$ & 251.02 & $\mathbf{a}_{1}$ & {$[0.968,-0.111,0.223]^{\mathrm{T}}$} \\
\hline & & & $\lambda_{2}$ & 73.80 & $\mathbf{a}_{2}$ & {$[-0.244,-0.245,0.938]^{\mathrm{T}}$} \\
\hline & & & $\lambda_{3}$ & 27.94 & $\mathbf{a}_{3}$ & {$[0.05,0.963,0.265]^{\mathrm{T}}$} \\
\hline \multirow{3}{*}{ Millet } & \multirow{3}{*}{$\overline{\mathbf{c}}$} & \multirow{3}{*}[47.715,-3.034,16.218]{$^{\mathrm{T}}$} & $\lambda_{1}$ & 184.03 & $\mathbf{a}_{1}$ & {$[0.895,-0.153,0.419]^{\mathrm{T}}$} \\
\hline & & & $\lambda_{2}$ & 50.83 & $\mathbf{a}_{2}$ & {$[-0.446,-0.347,0.825]^{\mathrm{T}}$} \\
\hline & & & $\lambda_{3}$ & 15.99 & $\mathbf{a}_{3}$ & {$[-0.019,0.925,0.379]^{\mathrm{T}}$} \\
\hline \multirow{3}{*}{ Monet } & \multirow{3}{*}{$\overline{\mathbf{c}}$} & \multirow{3}{*}[59.565,-4.118,8.383]{$^{\mathrm{T}}$} & $\lambda_{1}$ & 118.47 & $\mathbf{a}_{1}$ & {$[0.647,-0.238,0.724]^{\mathrm{T}}$} \\
\hline & & & $\lambda_{2}$ & 104.78 & $\mathbf{a}_{2}$ & {$[-0.763,-0.197,0.616]^{\mathrm{T}}$} \\
\hline & & & $\lambda_{3}$ & 23.41 & $\mathbf{a}_{3}$ & {$[0.004,0.951,0.309]^{\mathrm{T}}$} \\
\hline \multirow{3}{*}{ Renoir } & \multirow{3}{*}{$\overline{\mathbf{c}}$} & \multirow{3}{*}[52.161,-1.292,12.023]{$^{\mathrm{T}}$} & $\lambda_{1}$ & 166.44 & $\mathbf{a}_{1}$ & {$[0.816,-0.085,0.571]^{\mathrm{T}}$} \\
\hline & & & $\lambda_{2}$ & 73.68 & $\mathbf{a}_{2}$ & {$[-0.569,0.047,0.821]^{\mathrm{T}}$} \\
\hline & & & $\lambda_{3}$ & 47.32 & $\mathbf{a}_{3}$ & {$[0.097,0.995,0.01]^{\mathrm{T}}$} \\
\hline
\end{tabular}

space, which has the average color with $\mathrm{L}^{*}=54.72$ and the principal direction with positive $\mathrm{L}^{*}$ and $\mathrm{b}^{*}$. In addition, many colors are used because of the large deviations $\lambda_{1}=409.76$ and $\lambda_{2}=143.62$. Therefore, the color features of van Gogh tend to be bright and colorful. Next consider Millet's color features. Millet's ellipsoid is placed at a lower position and thin. The average color has $\mathrm{L}^{*}=47.71$ and the principal axis is directional toward only positive $L^{*}$. In addition, we note the relation $\lambda_{1}(=184.03) \gg \lambda_{2}>\lambda_{3}$. These indicate that his paintings 
have a small color contrast, and the color choices are less colorful and relatively dark in his paintings.

The color features of the six artists determined in this experiment are summarized as follows:

(1) Cezanne: moderate bright, yellow-greenish

(2) van Gogh: bright, colorful

(3) Manet: dark, less colorful, dull

(4) Millet: dark, less colorful, small color contrast

(5) Monet: bright, color distribution similar to Cezanne

(6) Renoir: colorful, warm

\subsection{Validation with profession visual assessment}

We performed a human visual assessment experiment using all printed color images for all artists in order to validate the artists' color features extracted from the image data. Figure 9 shows the scene of visual assessment. All color images of the art paintings were printed by an EPSON inkjet printer (PG-930) calibrated to match color with sRGB under D65. The evaluation items were "bright-dark", "colorful-less colorful", "vividdull", and "warm-cold". Twenty one evaluators consisting of one professor of art painting, one professor of image quality, one professor of national museum, and three professors, three researchers, and twelve graduate students of color imaging science and technology participated in the evaluation. These evaluators examined all the reproduced color images. The subjective impression of color features was compared among the image sets for different artists, and the artist's color features were ranked according to the evaluation items. The rank was determined on a 4-Likert scale, 'Extremely', 'Quite', 'Slightly', and 'Neutral'.

Figure 10 shows the visual assessment results for the paintings of the six artists. The vertical coordinates of each line in the figure represents the average ranking of all evaluators. The unique color features inherent to the respective artists are identified from the ranking results. For instance, van Gogh's paintings are evaluated to be slightly bright, colorful, and quite vivid. In contrast, Millet's paintings are evaluated to be quite dark, moderately less colorful, and quite dull. Note that these evaluations correspond to the results (2) and (4) shown in the previous subsection. Similarly, the human visual evaluations for the other artists are close to the previous results. Thus, it is suggested that the color features obtained from the PCA of image data of the respective artists' paintings are effective and valid to represent the color features of artists' paintings.

\section{Color Correction Algorithm for Color Mismatch Images}

\subsection{Basis algorithm}

The color correction is based on the coordinate transformation of pixel values in a color mismatch image. To

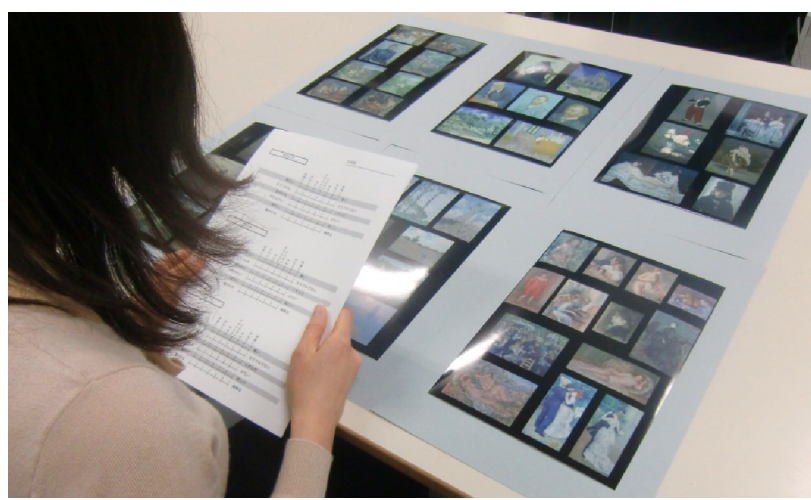

Figure 9 Scene of visual assessment

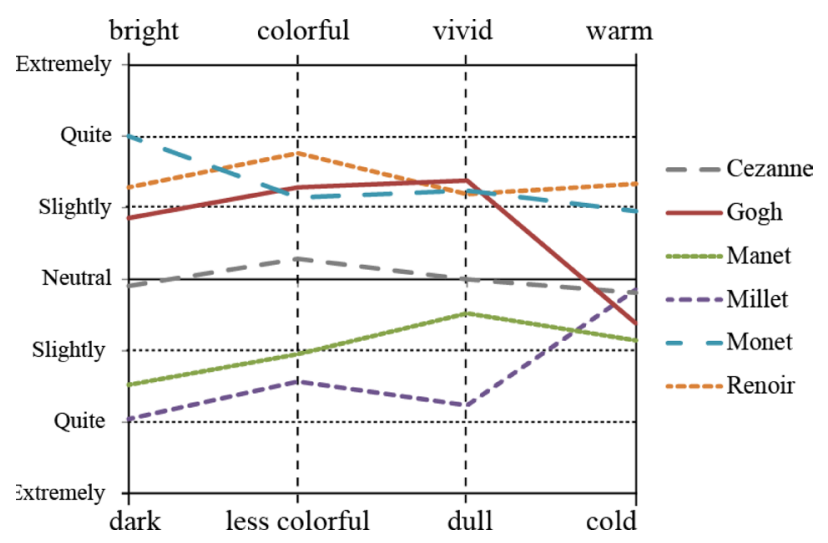

Figure 10 Results of the visual assessment experiment for seven artists' paintings

do so, the color features of the mismatched image are fitted to the artist's color features in the standard image database. Figure 11 shows the basic principle. The ellipsoid representing mismatch features is transformed to the ellipsoid of standard features by translation, scale, and rotation of the coordinate system. This transformation is applied to all pixel values of the color mismatch image.

First, we calculate the average color vector $\overline{\mathbf{c}}_{\mathrm{M}}$, the eigenvalues $\left(\lambda_{\mathrm{M} 1}, \lambda_{\mathrm{M} 2}, \lambda_{\mathrm{M} 3}\right)$, and the eivenvectors $\left(\mathbf{a}_{\mathrm{M} 1}\right.$, $\mathbf{a}_{\mathrm{M} 2}, \mathbf{a}_{\mathrm{M} 3}$ ) from a color mismatch image. We define a $3 \times 3$ eigen matrix $\mathbf{A}_{\mathrm{M}}$ for the color mismatch image as. $\left(\mathbf{A}_{\mathrm{M}}=\left[\mathbf{a}_{\mathrm{M} 1}, \mathbf{a}_{\mathrm{M} 2}, \mathbf{a}_{\mathrm{M} 3}\right]\right)$ Next, let $\overline{\mathbf{c}}_{\mathrm{S}},\left(\lambda_{\mathrm{S} 1}, \lambda_{\mathrm{S} 2}, \lambda_{\mathrm{S} 3}\right)$, and $\mathbf{A}_{\mathrm{S}}$ $\left(=\left[\mathbf{a}_{\mathrm{S}}, \mathbf{a}_{\mathrm{S} 2}, \mathbf{a}_{\mathrm{S} 3}\right]\right)$ be the mean color vector, the eigenvalues, and the eigen matrix for the standard image dataset, respectively. The color vector $\mathbf{c}_{\mathrm{M}}\left(=[R, G, B]^{T}\right)$ of the color mismatch image is corrected by the transformation

$$
\mathbf{c}^{\prime}=\overline{\mathbf{c}}_{\mathrm{S}}+\mathbf{A}_{\mathrm{S}}^{-1} \Lambda \mathbf{A}_{\mathrm{M}}\left(\mathbf{c}_{\mathrm{M}}-\overline{\mathbf{c}}_{\mathrm{M}}\right),
$$

Where $\Lambda$ is a scaling matrix defined by

$$
\Lambda=\left[\begin{array}{ccc}
\sqrt{\lambda_{\mathrm{S} 1} / \lambda_{\mathrm{M} 1}} & 0 & 0 \\
0 & \sqrt{\lambda_{\mathrm{S} 2} / \lambda_{\mathrm{M} 2}} & 0 \\
0 & 0 & \sqrt{\lambda_{\mathrm{S} 3} / \lambda_{\mathrm{M} 3}}
\end{array}\right] .
$$




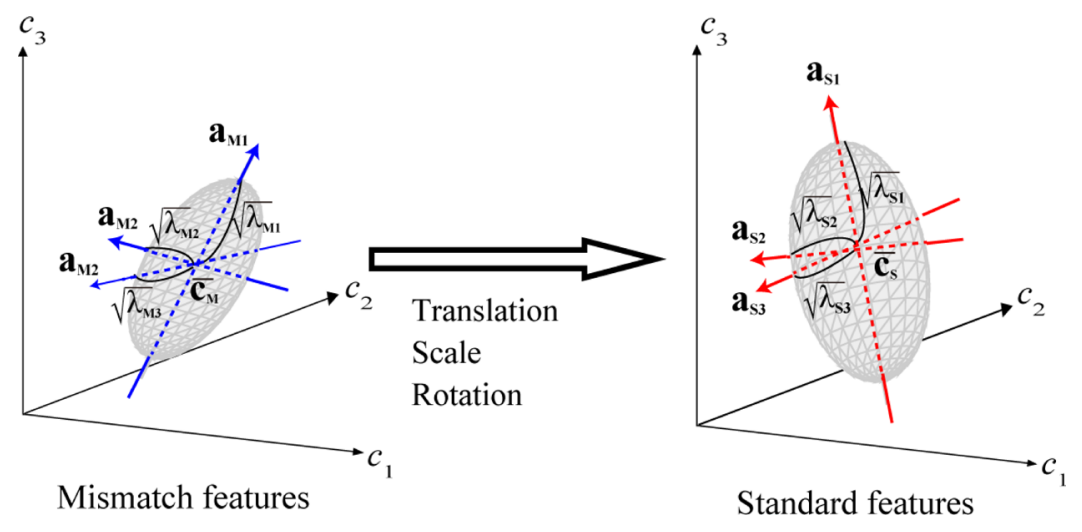

Figure 11 Basic principle of color correction

The above transformation is composed of three basic functions of (1) average matching by translating $\overline{\mathbf{c}}_{\mathrm{M}}$ to $\overline{\mathbf{c}}_{S}$, (2) axes matching by rotating the principal components $\mathbf{A}_{\mathrm{M}}$ to $\mathbf{A}_{\mathrm{S}}$, and (3) variance matching by scaling the color distribution by $\Lambda$. This transformation looks similar to that of scene color transfer ${ }^{6}$. However, the algorithm for scene color transfer did not include the translation step. This translation step is crucial in the present problem because the center of gravity of color distribution has a high influence in the reproduced color images.

\subsection{Algorithm in the CIELAB color space}

The merit of using the CIELAB is that color correction is done in a perceptually uniform color space. In other words, it is expected that the color-mismatch image of a painting is corrected closely to human visual evaluation. Let us compare the artists' ellipsoids in Figure 8 to those in Figure 7. The ellipsoids in the CIELAB look fatter, like rugby balls. On the other hand, those in the sRGB are thinner and more linear. The color distribution constructs a compact linear cluster, while the color histogram of the same painting exhibits a more diffused color distribution in the CIELAB.

Therefore, we consider an additional computation to the above basic algorithm. In order to suppress the occurrence of false colors by rotation and scale, we emphasize the color transformation to pixels far from the white point $\left(L^{*}, a^{*}, b^{*}\right)=(100,0,0)$, and emphasize less the transformation to pixels close to the original white point. This is because human vision is sensitive for the white area in paintings, and the color-mismatch images, acquired from picture collection books or through the internet, often differ in color from the original but keep the original white. Therefore, the additional computation is described as a weighted average

$$
\mathbf{c}^{\prime \prime}=w \mathbf{c}^{\prime}+(1-w) \mathbf{c}_{\mathrm{M}} \text {. }
$$

Here the weighting coefficient $\mathrm{w}$ is defined as a distance function from the white point $\mathbf{c}_{\mathrm{W}}=[100,0,0]^{T}$ as

$$
w=\left|\mathbf{c}_{\mathrm{M}}-\mathbf{c}_{\mathrm{W}}\right| / D
$$

where a constant $D$ is determined empirically. Normally $D$ is selected as the maximal distance from the white point to pixel color coordinates in the color space. In our experiments, we adopt $D=100$ as the distance between black and white points.

\section{Color image rendering in target scene \\ 4.1 Color temperature estimation of scene illumi- nant}

The painting images corrected under D65 Illuminant are further transformed for rendering more realistic color appearance in a favorite illumination condition. The first stage is to estimate illuminant in a target scene. We use the sensor correlation method ${ }^{7}$ to estimate the color temperature of scene illuminant. This method is effective to classify scene illuminant by the color temperature of blackbody radiators. The illuminant gamuts are created in advance by using a database of surface-spectral reflectance and the illuminants of blackbody radiators at different color temperatures. We calculate correlation values between the image color gamut determined from the camera sensor outputs and the reference illuminant gamuts. Then, the correlated color temperature of scene illuminant is estimated by the illuminant gamut with the maximum correlation value.

Figure 12 shows the illuminant gamuts defined in the $(\mathrm{R}, \mathrm{B})$ sensor plane of the digital camera used in this paper. The gamuts are depicted at the reciprocal color temperatures, spanning from 118 mired $(8500 \mathrm{~K})$ to 400 mired $(2500 \mathrm{~K})$ in 11.5 increments. The image color gamut under unknown illuminant is specified by a convex hull of the observed pixel distribution on the $(\mathrm{R}, \mathrm{B})$ plane. A correlation value is then computed by

$$
r_{i}=G_{I i} / \sqrt{G_{I} G_{i}},
$$

where $G_{I}$ and $G_{i}$ are the areas of an image and the i-th illuminant gamuts, respectively, and $G_{I i}$ is the area of over- 


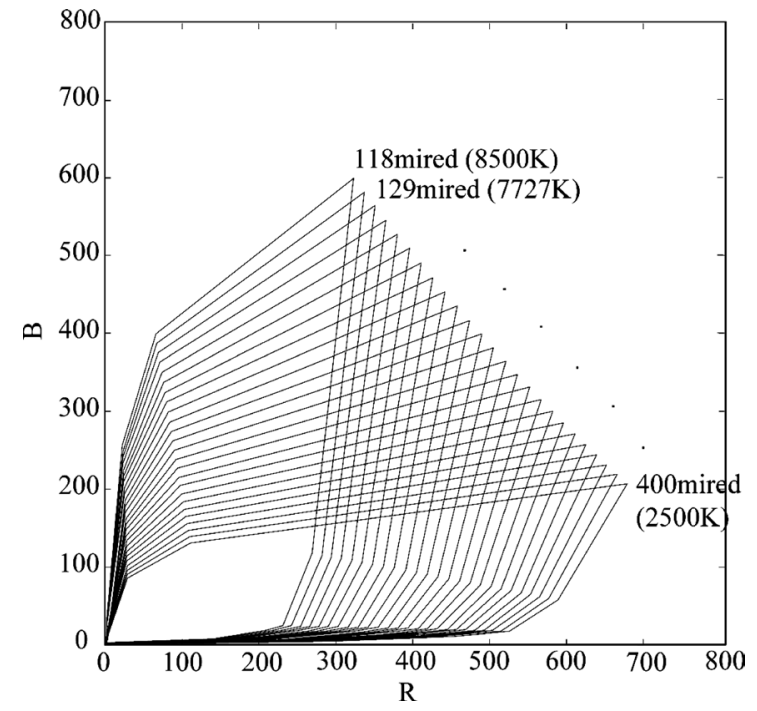

Figure 12 Illuminant gamuts for blackbody radiators in the $(R, B)$ sensor plane of the digital camera used in this paper

lap between the image gamut and the illuminant gamut.

If there is large intensity difference between images, the image data are adjusted by a simple scaling operation, equivalent to the adjusting of the exposure duration. To scale the data, we denote the pixel intensity by

$$
I=\left(R^{2}+G^{2}+B^{2}\right)^{1 / 2}
$$

and let $I_{\max }$ be the maximum intensity over the image. The normalized RGB values are calculated as

$$
(R, G, B)=\left(k R / I_{\max }, k G / I_{\max }, k B / I_{\max }\right)
$$

where $k$ is the most proper scaling parameter used to adjust the data.

\subsection{Color image transformation to target illuminant}

The image of an art printing under a target illuminant condition is rendered using the estimated correlated color temperature. We present the image in terms of the tristimulus values $\mathrm{XYZ}$ for computational convenience. The color values of each pixel are then transformed by taking the chromatic adaptation effect into account. We use a von Kries-type transformation.

First, all XYZ values of the image are transformed to relative cone responses LMS using a linear matrix multiplication [8]

$$
\left[\begin{array}{c}
L \\
M \\
S
\end{array}\right]=\mathbf{M}\left[\begin{array}{c}
X \\
Y \\
Z
\end{array}\right],
$$

where

$$
\mathbf{M}=\left[\begin{array}{rrr}
0.400 & 0.708 & -0.081 \\
-0.226 & 1.165 & 0.046 \\
0.000 & 0.000 & 0.918
\end{array}\right] .
$$

Second, we calculate the cone responses for white under the standard D65 Illuminant and the cone responses under the target illuminant based on the estimated color temperature. The gain coefficients of chromatic adaptation are calculated as the ratio of LMS values as

$$
w_{\mathrm{L}}=L_{\mathrm{T}} / L_{\mathrm{S}}, \quad w_{\mathrm{M}}=M_{\mathrm{T}} / M_{\mathrm{S}}, \quad w_{\mathrm{S}}=S_{\mathrm{T}} / S_{\mathrm{S}} .
$$

We assume complete chromatic adaptation. Then, the $\mathrm{XYZ}$ image under the target illuminant is obtained from a matrix equation

$$
\left[\begin{array}{c}
X^{\prime} \\
Y^{\prime} \\
Z^{\prime}
\end{array}\right]=\mathbf{M}^{-1}\left[\begin{array}{ccc}
w_{\mathrm{L}} & 0 & 0 \\
0 & w_{\mathrm{M}} & 0 \\
0 & 0 & w_{\mathrm{S}}
\end{array}\right] \mathbf{M}\left[\begin{array}{c}
X \\
Y \\
Z
\end{array}\right] .
$$

Finally, the XYZ image is further transformed into the RGB image for a display device.

\section{Experimental results}

\subsection{Acquisition of color-mismatch images}

Samples of color mismatch images for the famous oil paintings shown in 2.1 were collected in two ways using a scanner and the internet. One set of painting images was acquired from the scanned pictures in three painting collection books ${ }^{9-11)}$ by using an HP scanner (Scanjet G4050) in the resolution of $200 \mathrm{dpi}$. Another set was acquired from the museum pictures in two websites through the internet ${ }^{1213)}$. Figure 13 demonstrates three samples of color mismatch images for Monet's "The Fife Player." We notice that the colors of the sample images do not match each other at all.

\subsection{Color correction results}

The color correction algorithm was applied to the three images in Figure 13. The ellipsoids of Manet in Figure 7(c) and Figure 8(c), based on the six selected color images in the database, were used as the color features in the sRGB and CIELAB color spaces under D65. To evaluate if the proposed method is able to recover the exact color images, the color features were recomputed from the set of Manet's paintings, excluding the Fife Player.

The original painting of The Fife Player was photographed in the Orsay Museum by the imaging system in 2.2. The correlated color temperature of the illuminant was determined as $3151 \mathrm{~K}$ by using the white reference plate. The camera image was transformed to the standard image under D65 Illuminant by using the procedure in 2.3. Figure 14 shows the camera image and the standard image. Figures 15 and 16 show the correction results for the image in the sRGB color space by using the procedure in 3.1 and the CIELAB color space by using the procedure with $\mathrm{D}=100$ in 3.2 under $\mathrm{D} 65$ Illuminant. 


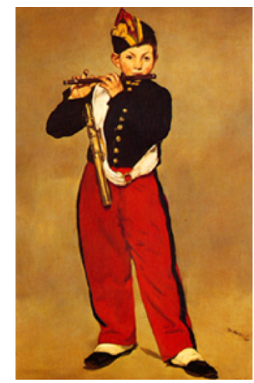

(a) Picture book $\mathrm{S}$

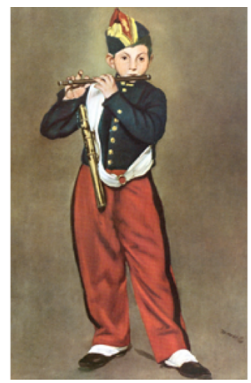

(b) Picture book N

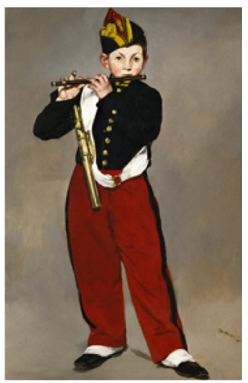

(c) Internet 1

Figure 13 Samples of color mismatch images for Monet's "The Fife Player"

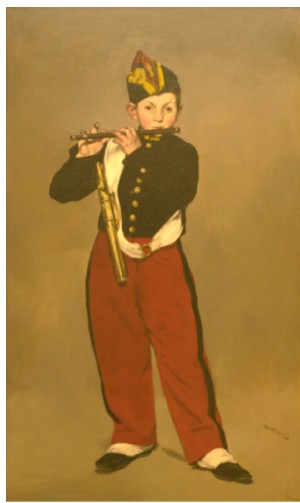

(a) Camera image

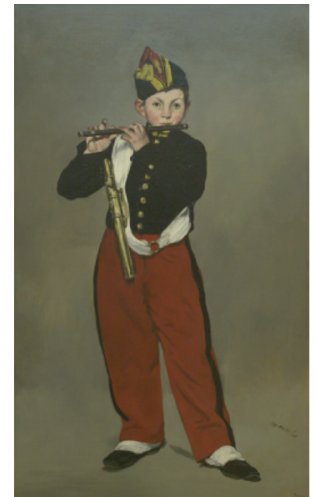

(b) Standard image (D65)

Figure 14 Camera image and standard image of the Fife Player by Manet

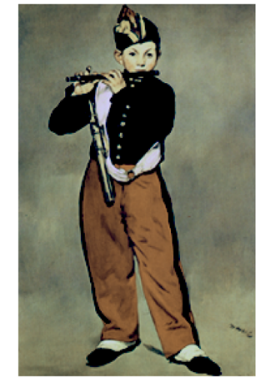

(a) Picture book $\mathrm{S}$

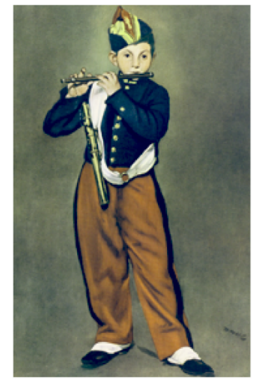

(b) Picture book N

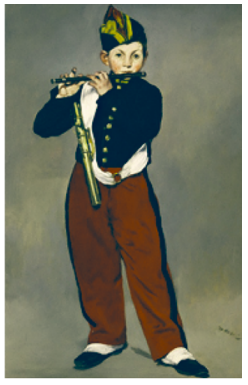

(c) Internet 1

Figure 15 Color correction results in the sRGB color space

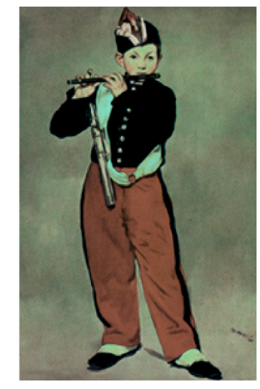

(a) Picture book $\mathrm{S}$

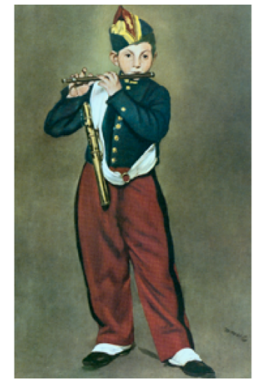

(b) Picture book N

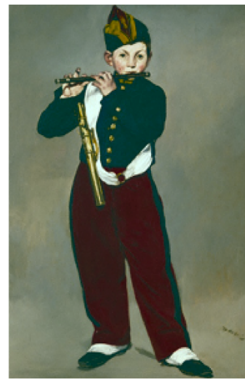

(c) Internet 1

Figure 16 Color correction results in the CIELAB color space 
For numerical evaluation, we calculated the color difference between the original image in Figure 13 and the standard color image in Figure 14(b), and also between the corrected color images in Figures 15, 16 and the standard color image. The CIE1976 color difference $\Delta L_{\mathrm{ab}}^{*}[14]$ is defined between $\left(L_{1}^{*}, a_{1}^{*}, b_{1}^{*}\right)$ and $\left(L_{2}^{*}, a_{2}^{*}, b_{2}^{*}\right)$ in the CIELAB color space as

$$
\Delta L_{a b}^{*}=\sqrt{\left(L_{1}^{*}-L_{2}^{*}\right)^{2}+\left(a_{1}^{*}-a_{2}^{*}\right)^{2}+\left(b_{1}^{*}-b_{2}^{*}\right)^{2}} .
$$

All color differences in the correction results for sRGB and CIELAB images are summarized in Table 2.

Table 2 Color differences $\Delta L_{a b}^{*}$ in color correction results for the images in Figure 14

\begin{tabular}{|c|c|c|c|}
\hline \multirow{2}{*}{ Sample } & \multirow{2}{*}{ Original $\Delta L_{a b}^{*}$} & \multicolumn{2}{|c|}{ Color correction } \\
\cline { 3 - 4 } & & $\Delta L_{a b}^{*}$ for sRGB image & $\Delta L_{a b}^{*}$ for LAB image \\
\hline (a) & 42.5 & 13.6 & 12.9 \\
\hline (b) & 14.9 & 14.3 & 11.2 \\
\hline (c) & 14.1 & 12.1 & 12.1 \\
\hline
\end{tabular}

It was found that all color-mismatch images improved in the sense of numerical color difference. The biggest difference in color appearance observed in Figure 13(a) was found to be greatly improved, and the corrected images in Figures 15(a) and 16(a) are closer to the exact color of the image on Figure 14(b).

\subsection{Image rendering results in target scene}

Figure 17(a) shows a target scene for color image rendering. It represents the scene of the painting of Lola of Valance by Manet in the Orsay Museum. We suppose that the painting of the Fife Player is hung on the wall in the exhibition room. First, the color temperature of scene illuminant was estimated from the scene image in Figure 17(a), captured in the Orsay Museum. Figure 17(b) depicts pixel distribution of the scene image with the illuminant gamuts in the $(\mathrm{R}, \mathrm{G})$ plane. According to the sensor correlation algorithm, the correlation value was computed between the image gamut and the illuminant gamuts with different color temperatures. The correlated color temperature was then estimated as

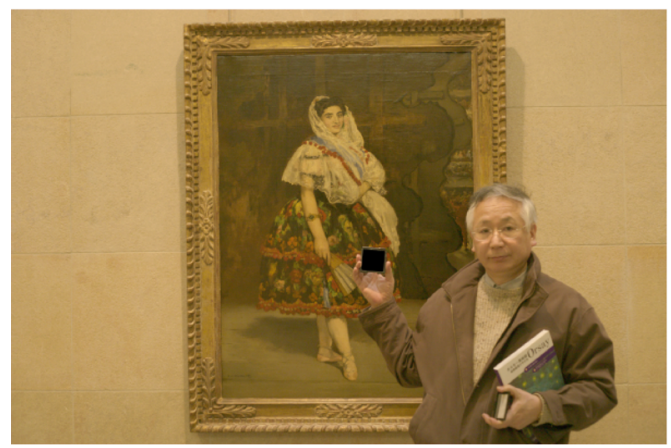

(a) Target scene

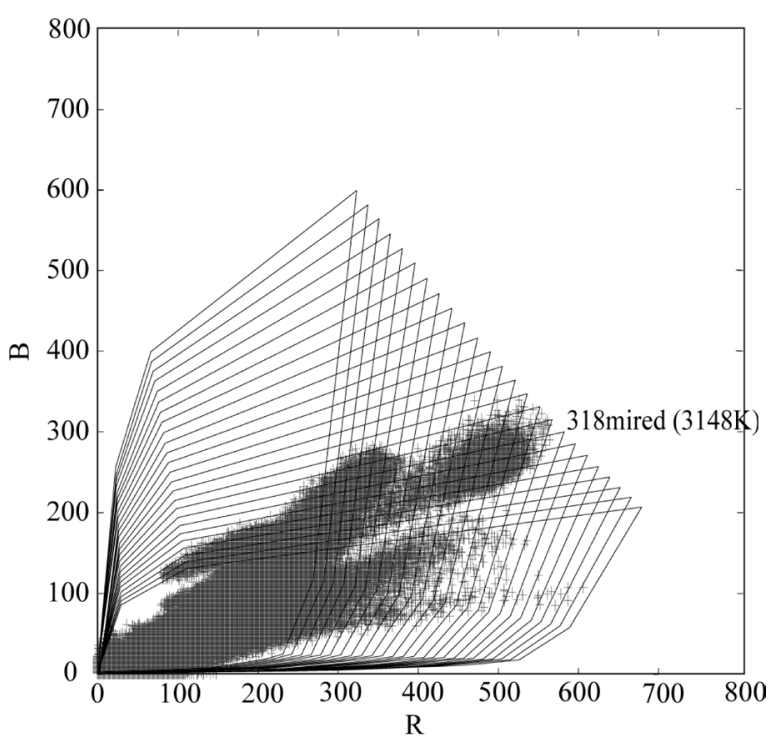

(b) Pixel distribution of the scene image

Figure 17 Target scene for color image rendering

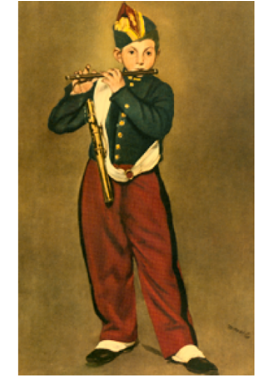

(a) Picture book $\mathrm{S}$

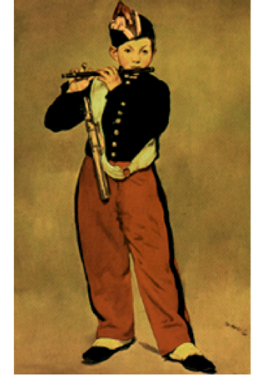

(b) Picture book $\mathrm{N}$

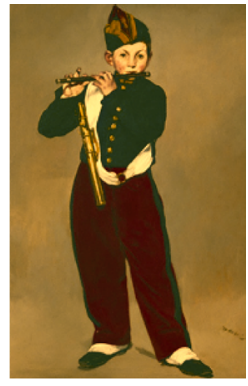

(c) Internet 1

Figure 18 Rendered color images under illuminant of the target scene 
$3148 \mathrm{~K}$ (318 mired). This estimate is fairly close to the direct measurement $3131 \mathrm{~K}$ by the white reference plate.

Finally, the painting images under D65 were further transformed on this illuminant condition. Figure 18 shows the rendered color images for the target scene, which were transformed from the corrected images in the CIELAB color space, shown in Figure 17.

Note that the estimated color temperature $3148 \mathrm{~K}$ of the target scene is almost coincident with the color temperature $3151 \mathrm{~K}$ of the original scene where the Fife Player was captured. Therefore, the validity of the rendered images can be seen by comparing the three images in Figure 18 to the original image in Figure 13(a).

The whole feasibility of the proposed method is confirmed with this experimental evidence.

\section{Conclusion}

The present paper has proposed a method to correct the color-mismatch images of paintings based on artists' color features, and to reproduce the exact color images under a specific illumination condition. First, we described a standard image database consisting of famous oil paintings we captured directly, and an algorithm for extracting the artists' color features based on the color distribution of the standard image data for each artist. The color distribution was analyzed by PCA and described with an ellipsoid to represent the standard color features for the artist. It was suggested that the color features for the respective artists were similar to the human visual assessment of their paintings. Next, the color correction was based on the coordinate transformation of pixel values in a color-mismatch image so that the color features of the mismatched image were fitted to the artist's color features in the standard image database. We presented the correction algorithms using the two color spaces of the RGB and the CIELAB. Moreover, we described a procedure for rendering the exact color images under a favorite scene illuminant condition. The color temperature of the scene illuminant was estimated from the camera sensor outputs and the color image under D65 was further transformed to a target image based on the estimated color temperature. Experiments were performed using samples of colormismatch images of the famous oil paintings, which were collected from painting picture books and the internet. The feasibility of the proposed method was confirm- ed on the evidences of the correction results of the mismatched images. In our future research, we will improve the method to reduce the color differences in correcting the mismatched images.

\section{References}

(1) Martinez, K., Cupitt, J., Saunders, D. and Pillay, R.: Ten years of art painting research, Proc. of The IEEE, 90 (1) pp. 28-41 (2002).

(2) Lombardi, T. E.: The classification of style in fineart painting, ETD Collection for Pace University, Paper AAI3189084 (2005).

(3) Saito, R., Horiuchi, T. and Tominaga, S.: Feature extraction of painter-specific color distribution from real paintings, Proc. Congress of the International Colour Association (AIC 2010), Mar del plata, pp. 600-603 (2010).

(4) Tominaga, S., Ishida, A. and Wandell, B. A.: Color temperature estimation of scene illumination by the sensor correlation method, IEICE, Vol. J85-D-II, No. 5, pp. 1180-1189 (2002) (In Japanese).

(5) IEC 61966-2-1, Title Multimedia systems and equipment-Colour measurement and management-Part 2-1: Colour management-Default RGB colour space-sRGB (1999).

(6) Kotera, H.: A Scene-Referred Color Transfer for Pleasant Imaging on Display, Proc. of IEEE International Conference on Image Processing (ICIP2005), Genova, Italy, Vol. 2, pp. 11-14 (2005).

(7) Tominaga S. and Wandell, B. A.: Natural scene illuminant estimation using the sensor correlation, Proceedings of The IEEE, 90 (1), pp. 42-56 (2002).

(8) Fairchild, M. D.: Color Appearance Models, AddisonWesley (1998).

(9) Abe, Y.: Manet Nippon Bunko Art Shincho 24, Shinchosha (1974) (In Japanese).

(10) Richardson, J.: Manet Art Library, Nishimurashoten (1999) (In Japanese).

(11) Kilian, D.: A fuller understanding of the paintings at Orsay, Artlys (2001).

(12) http://www2.edu.ipa.go.jp/gz/index.html

(13) http://art.pro.tok2.com/

(14) Wyszecki G. and Stiles, W.S.: Color Science: Concepts and Methods, Quantitative Data and Formulae, Chapter 3, John Wiley \& Sons (1982). 